\title{
Estudo da dinâmica da paisagem na bacia hidrográfica do Arroio Bocarra, Bagé, RS
}

\author{
Study of landscape dynamics in the Bocarra River watershed, Bagé, RS
}

\author{
Alexandro Gularte Schafer '
}

\section{RESUMO}

O bioma Pampa vem experimentando um acelerado processo de alteração no uso e cobertura da terra nas últimas décadas que exerce influência tanto nos padrões de paisagem quanto nos recursos hídricos. O objetivo deste estudo foi mapear as mudanças que ocorreram no uso e cobertura da terra na bacia hidrográfica do Arroio Bocarra (RS) e avaliar a sua influência nos padrões de paisagem entre os anos de 1985 a 2017. Para tanto, utilizou-se uma série temporal de imagens de satélite (1985, 1996, 2003 e 2017), modelos matemáticos e métricas de paisagem. Os resultados indicam que houve consideráveis mudanças no uso e cobertura da terra na bacia hidrográfica nos últimos trinta e dois anos, que impactaram os índices relacionados à paisagem. $\mathrm{A}$ taxa de mudança global obtida a partir do índice de estado e tendência das mudanças $\left(P_{t}\right)$ foi de 0,74 , indicando transição em uma via, dominada pela conversão de áreas campestres por áreas destinadas a agricultura, ocasionada pela expansão do cultivo de soja na região ao longo dos últimos anos. A diversidade da paisagem aumentou de 0,86 em 1985 para 1,07 em 2017, enquanto a dominância diminuiu de 0,93 para 0,72, o que indica que houve uma redução nas diferenças entre os padrões de paisagem. A fragmentação da paisagem reduziu entre 1985 a 2017, enquanto a área média dos fragmentos aumentou.

Palavras-chave: Recursos hídricos; Métricas da paisagem; Dinâmica temporal

\section{ABSTRACT}

The Pampa biome is experiencing an accelerated Land Use and Land Cover Change process in the last decades that influence both the landscape patterns and water resources. The aim of this study was to map the land use and land cover changes in the Bocarra River watershed and to evaluate their influence on landscape patterns from 1985 to 2017. Therefore, satellite images series (1985, 1996, 2003 and 2017), mathematical models and landscape metrics were used. The results indicate that there have been considerable changes in land use and land cover in the watershed over the past thirty-two years, which impacted landscape-related indices. The global rate of change obtained from the status and trend in land change index (Pt) was 0.74 , indicating a oneway transition dominated by the conversion of grasslands to croplands over time, which was caused by the expansion of soy cultivation in the region. Landscape diversity increased from 0.86 in 1985 to 1.07 in 2017, while dominance decreased from 0.93 to 0.72 , indicating that there was a reduction in differences between landscape patterns. Landscape fragmentation decreased from 1985 to 2017, while the average area of fragments increased.

Engenheiro Civil. Mestrado em Engenharia Civil, área de cadastro técnico, trabalho desenvolvido na área de Sensoriamento Remoto aplicado à engenharia. Doutorando do PPGEC-UFSC, área de gestão territorial. E-mail: alschafer@gmail.com ORCID: http://orcid.org/0000-0001-8700-0860 


\section{INTRODUÇÃO}

Os ecossistemas terrestres são importantes componentes da natureza, exercendo influência na regulação climática e no ciclo hidrológico, além de constituir fonte de recursos naturais para as necessidades humanas (JIMÉNEZ et al., 2018). Ao longo das últimas décadas, registra-se uma intensificação na alteração dos ecossistemas em todo o planeta. As mudanças no uso e cobertura da terra e a intensificação no uso da terra são as principais causas por trás da degradação dos ecossistemas, sendo relacionados ainda a perdas na biodiversidade, ao esgotamento de serviços ecossistêmicos, às mudanças na paisagem (BEBI et al., 2017; PETZ, 2014; CARPIO et al., 2016), às alterações no estoque de carbono dos solos e às mudanças climáticas (TITEUX et al., 2016). As mudanças no uso e cobertura da terra refletem o inter-relacionamento entre atividades humanas/sociais/econômicas e os processos ecológicos naturais (CHEN et al., 2018), sendo um dos indicadores utilizados para determinar o impacto de atividades antropogênicas nos ecossistemas (LUO et al., 2018). As principais causas das mudanças no uso e cobertura da terra podem ser classificadas, segundo Eiter e Pothoff (2007), em: (a) Forças extrínsecas (socioeconômica, tecnológica, recreacional, legislação de proteção); (b) Forças intrínsecas indiretas (infraestrutura de transporte, agricultura sazonal, vegetação, turismo e recreação ao ar livre, proteção natural e da paisagem); e (c) forças intrínsecas diretas (força de trabalho, população, secas e supressão de áreas de gramíneas). Atualmente, os impactos da expansão urbana e agrícola e a homogeneização da paisagem ocorrem tanto em escala local quanto em escala global. No sul do Brasil, as alterações no uso e cobertura da terra vêm ocorrendo de forma rápida e intensa ao longo do último século e de maneira ainda mais acelerada a partir de 1950, com a expansão do agronegócio (FREITAS et al., 2018).

O entendimento da dinâmica do uso e da cobertura da terra vem sendo reconhecido como um elemento de grande relevância no âmbito das pesquisas em mudança ambiental (LAMBIN et al., 2003) e em sustentabilidade (YANG et al., 2016; LIU et al., 2018). O monitoramento e a quantificação das mudanças no uso e cobertura da terra são essenciais 
para alcançar um gerenciamento da terra adequado (JIMENEZ et al., 2018). A análise multitemporal da paisagem pode ser utilizada para entender os processos e os padrões da paisagem ao longo do tempo, verificando como eles se comportam e quantificando as taxas relacionadas à dinâmica das mudanças (LOPEZ-GRANADO et al., 2013). Nesse sentido, o cálculo de métricas da paisagem é amplamente usado para descrever os padrões de paisagem. Embora não exista, atualmente, um subconjunto de métricas padrão que seja amplamente aceito e utilizado para esse fim (WANG e YANG, 2012).

Os dados provenientes do sensoriamento remoto orbital vêm sendo utilizados para quantificar e monitorar as mudanças no uso e cobertura da terra, a fim de entendê-las com relação às alterações climáticas e às atividades humanas (DUAN et al., 2019). As imagens de satélite do programa Landsat, que abriga o mais longo registro continuo de imagens de moderada resolução da superfície terrestre em nível global, são comumente utilizadas para identificar tendências no uso da terra. (KANG et al., 2019). A aplicação integrada de sensoriamento remoto e de Sistemas de Informações Geográficas (SIG) provou ser efetiva no entendimento das taxas de mudança do uso e cobertura da terra no tempo e no espaço (BAIDYA et al., 2009; WANG et al., 2011),. Os estudos de detecção de mudanças com base em sensoriamento remoto normalmente têm como foco inicial proporcionar quantificações de quanto, onde e que tipo de mudanças vêm ocorrendo (FOODY, 2002; ALEMAYEHU et al, 2009).

Este artigo tem como objetivo geral investigar a dinâmica das mudanças no uso e cobertura da terra e seus efeitos nos padrões de paisagem na bacia hidrográfica do Arroio Bocarra, localizada no município de Bagé, Rio Grande do Sul, em um período de trinta e dois anos. Para tanto, integrou-se métodos de ecologia da paisagem, sensoriamento remoto e sistemas de informações geográficas (SIG), com base em uma série histórica de imagens dos sensores TM e OLI (Landsat) Landsat TM e OLI (imageadas em 1985, 1996, 2003 e 2017).

\section{2 ÁREA DE ESTUDO}

A bacia hidrográfica do Arroio Bocarra (Figura 1) faz parte da bacia hidrográfica do Rio Negro, RS e está localizada entre as coordenadas $31^{\circ} 18^{\prime} 07^{\prime \prime}$ e $31^{\circ} 31^{\prime} 52^{\prime \prime}$ sul e $54^{\circ} 18^{\prime}$ 
$54^{\prime \prime}$ e $54^{\circ} 20^{\prime} 31^{\prime \prime}$ oeste. A bacia está completamente inserida no município de Bagé, RS e tem área de drenagem de aproximadamente $215,00 \mathrm{~km}^{2}$. O Arroio Bocarra é um dos principais afluentes do Rio Negro, Rio Grande do Sul. As altitudes na bacia hidrográfica variam de $139 \mathrm{~m}$ a $314 \mathrm{~m}$.

Figura 1 - Localização da bacia hidrográfica do Arroio Bocarra. Organização: O autor, 2020.

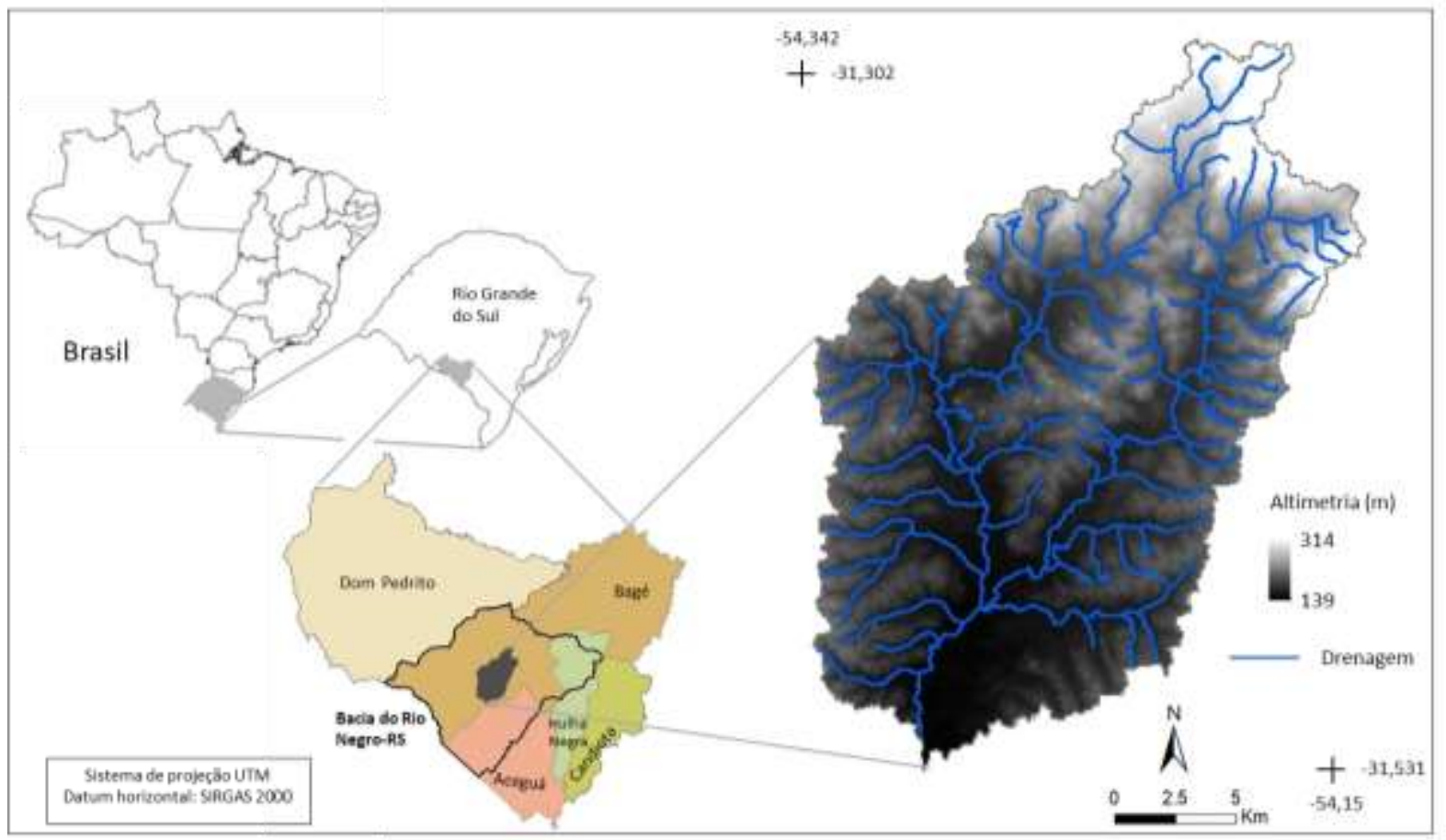

A região onde a bacia hidrográfica está inserida apresenta, segundo a classificação de Köeppen, clima subtropical ou virginiano (Cfa), pertencente à área morfoclimática denominada Escudo Sul-Riograndense-Uruguaio. As temperaturas médias máximas giram em torno de $24,1^{\circ} \mathrm{C}$ e as médias mínimas em torno de $13,1^{\circ} \mathrm{C}$. Os solos do tipo Brunizem e laterítico abrangem a maior parte da bacia. A precipitação média anual em Bagé fica em torno de $1.423 \mathrm{~mm}$, sendo que ocorrem períodos de estiagem em que a precipitação mensal chega a ser nula, principalmente no período de novembro a março, como vem sendo registrado nos últimos anos (DAEB, 2007). 


\section{MATERIAIS E MÉTODOS}

Os métodos de pesquisa podem ser agrupados nas seguintes etapas: 1) aquisição de dados primários e mapeamento do uso e cobertura da terra; 2) cálculo de métricas da paisagem e caracterização da mudança na paisagem; e 3) verificação do estado e da tendência das mudanças no uso e cobertura da terra.

\subsection{Aquisição de dados primários e classificação do uso e cobertura da terra}

Os dados de uso e cobertura da terra foram obtidos a partir da classificação supervisionada de quatro imagens Landsat (sensor TM para as imagens de 1985, 1996 e 2003 e sensor OLI para a imagem de 2017), usando o método da Máxima Verossimilhança (MaxVer) no programa computacional ENVI, além de um mapa topográfico na escala 1:50.000, para a correção geométrica das imagens. A retificação geométrica e a normalização radiométrica foram realizadas na etapa de pré-processamento das imagens. Na Quadro 1, apresenta-se a chave de interpretação para as classes de uso e cobertura da terra, que foram definidas de acordo com IBGE (2013).

\section{Quadro 1 - Chave de interpretação de imagens}

\begin{tabular}{|c|c|c|c|c|}
\hline $\begin{array}{l}\text { Classe } \\
\text { Nível I }\end{array}$ & $\begin{array}{l}\text { Classe } \\
\text { Nível II }\end{array}$ & Coloração & $\begin{array}{l}\text { Amostra } \\
\text { 4R3G2B }\end{array}$ & Verdade terrestre \\
\hline $\begin{array}{c}\text { Área } \\
\text { urbanizada }\end{array}$ & $\begin{array}{c}\text { Área antrópica } \\
\text { não agrícola }\end{array}$ & Branco & & \\
\hline $\begin{array}{l}\text { Cultura } \\
\text { temporária }\end{array}$ & $\begin{array}{l}\text { Área antrópica } \\
\text { agrícola }\end{array}$ & $\begin{array}{c}\text { Tons de rosa e } \\
\text { Branco }\end{array}$ & & \\
\hline Silvicultura & $\begin{array}{l}\text { Área antrópica } \\
\text { agrícola }\end{array}$ & Preto & & \\
\hline Campestre & $\begin{array}{c}\text { Área de } \\
\text { vegetação } \\
\text { natural }\end{array}$ & Verde claro & & \\
\hline
\end{tabular}




\begin{tabular}{|c|c|c|c|c|}
\hline Área florestal & $\begin{array}{c}\text { Área de } \\
\text { vegetação } \\
\text { natural }\end{array}$ & Verde escuro & Água & $\begin{array}{c}\text { Azul escuro e } \\
\text { tons de roxo } \\
\text { continental }\end{array}$
\end{tabular}

Fonte: Organização dos autores

A próxima etapa metodológica foi a realização de pós-classificação, visando minimizar os erros identificados durante as saídas de campo e em consultas a imagens de alta resolução disponíveis no Google Earth. O mapeamento do uso e da cobertura da terra foi validado através da análise qualitativa e quantitativa dos dados gerados após essa etapa. $\mathrm{Na}$ análise qualitativa, foi realizada a comparação entre o mapa temático oriundo da classificação e as verdades terrestres, juntamente com o conhecimento adquirido nas saídas de campo. Para a imagem de 2003, utilizaram-se imagens de alta resolução disponíveis no Google Earth. Na análise quantitativa, foi considerado o coeficiente Kappa, índice que quantifica a exatidão do mapeamento a partir de uma escala proposta por Landis e Koch (1977). Os coeficientes Kappa obtidos após a etapa de pós-classificação foram de 0,851 para 1985, 0,882 para 1996, 0,874 para 2003 e 0,931 para 2017. As mudanças no uso e cobertura da terra foram identificadas para as seis classes, a partir de operações espaciais entre as imagens matriciais, utilizando programa computacional ArcMAP.

\subsection{Cálculo das métricas da paisagem}

As métricas da paisagem utilizadas no estudo foram: a) percentagem da paisagem correspondente a cada classe de uso e cobertura da terra; b) índice de diversidade; c) índice de dominância; d) número de fragmentos; e) índice de fragmentação; e f) área média dos fragmentos. Os itens $a, d$ e $f$ foram calculados no programa computacional FRAGSTATS 2.0 (MCGARIGAL et al., 2012) à partir dos arquivos de uso e cobertura da terra em formato matricial obtidos na etapa anterior. Esse programa calcula os índices de paisagem em nível de fragmento (elementos básicos do mosaico), classe (de uso e cobertura da terra) e paisagem (o mosaico de fragmentos como uma unidade completa) (TEIXIDO et al., 2002). 
Os índices de diversidade $(H)$, de dominância (D) e de fragmentação $(F)$ da paisagem foram calculados a partir das equações abaixo, em uma planilha eletrônica.

$$
\begin{array}{ll}
H^{\prime}=-\sum_{i=1}^{n} P_{i} \ln P_{i} & \text { Equação 1 } \\
H_{\text {max }}=\ln m & \text { Equação 2 } \\
D=H_{\text {max }}-H^{\prime} & \text { Equação 3 } \\
F=\left(N_{p}-1\right) / N_{c} & \text { Equação 4 }
\end{array}
$$

Onde $H^{\prime}$ é o índice de diversidade da paisagem, $P_{i}$ é a fração de área ocupada pela classe de uso e cobertura da terra, $n$ é o número total de classes. $H_{\max }$ é o índice máximo de diversidade da paisagem e o termo ln (logaritmo natural) $m$ representa a diversidade máxima. $D$ é o índice de dominância da paisagem, definido como a discrepância entre a diversidade em um determinado cenário $\left(H^{\prime}\right)$ e a diversidade máxima da paisagem $\left(H_{\max }\right)$ (O’NEILL et al., 1988). F é o índice de fragmentação, calculado a partir de $N_{p}$ (número total de classes de uso e cobertura da terra) e $N_{c}$ (relação entre a área total e a área mínima dos fragmentos), em que $0 \leq F \leq 1$.

O índice de diversidade da paisagem $(\mathrm{H})$ expressa o grau em que dada variedade de elementos de paisagem está representada na área em estudo em proporção igual. Quanto maior é o índice, mais diversa é a paisagem. Portanto, a diversidade máxima é alcançada quando todos os usos são representados em proporção igual. A aplicação do índice de dominância serve para medir a extensão da dominância de uma ou mais classes de uso e cobertura da terra. Valores pequenos para a dominância indicam que existem várias classes em proporções iguais na paisagem enquanto valores altos indicam que poucas classes dominam a cena (BRITALDO, 1998). O índice de fragmentação da paisagem (F) é usado para estimar a fragmentação da paisagem (O'NEILL et al., 1988), F=1 indica fragmentação total e $\mathrm{F}=0$ indica $a$ inexistência de fragmentação.

\subsection{Estado e tendência das mudanças do uso da terra}


As tendências das mudanças no uso e cobertura da terra podem ser descritas a partir da análise: a) de cada classe de uso e cobertura da terra, individualmente; e b) considerando todas as classes conjuntamente.

Para realizar as análises de classe de uso e cobertura da terra, é possível fazer uso dos índices de mudança $\left(N_{c}\right)$, de estado total de mudança $\left(T_{c}\right)$ e de direção e tendência $\left(P_{s}\right)$ (FENG et al., 2011; WANG E WANG, 2013). O índice $N_{c}$ é definido como a diferença entre o máximo e o mínimo de ganho ou de perda que ocorreu em cada classe de uso e cobertura da terra entre os cenários. $T_{C}$ é definido como a soma de ganho ou perda de uma área (PONTIUS et al. 2004, FENG et al. 2011, WANG e WANG, 2013) e reflete a dinâmica das mudanças no uso e cobertura da terra. Quatro tipos de estado podem ser atribuídos a uma classe de uso e cobertura da terra: balanceado (perdas iguais aos ganhos); quase balanceado (pequena desigualdade entre perdas e ganhos); desbalanceado (diferenças significantes entre perdas e ganhos); e extremamente desbalanceado (LUO et al., 2008). A direção pode ser definida tanto como uma transição em um único sentido quanto como uma transição em dois sentidos (duas vias). A tendência se refere à tendência de aumento ou de redução da ocupação de cada classe de uso e cobertura da terra (LUO et al. 2008; FENG et al. 2011; WANG e WANG, 2013). Assim, os índices $N_{c_{1}} T_{c_{1}}$ e $P_{s}$ são definidos como:

$$
\begin{array}{ll}
N_{c}=\left(U_{b}-U_{a}\right) \times 100 \% / U_{a}=\left(\Delta U_{\text {in }}-\Delta U_{\text {out }}\right) \times 100 \% / U_{a} & \text { Equação } 5 \\
T_{c}=\left(\Delta U_{\text {in }}+\Delta U_{\text {out }}\right) \times 100 \% / U_{a} & \text { Equação } 6 \\
P_{S}=\frac{N_{c}}{T_{c}}=\frac{\Delta U_{\text {in }}-\Delta U_{\text {out }}}{\Delta U_{\text {in }}+\Delta U_{\text {out }}}-1 \leq P_{S} \leq 1, \Delta U_{\text {in }}+\Delta U_{\text {out }} \neq 0 & \text { Equação } 7
\end{array}
$$

Em que $U_{a}$ e $U_{b}$ representam a área de uma classe de uso e cobertura da terra nos cenários "a" e "b", respectivamente. $\Delta \mathrm{U}_{\text {out }} \geq 0$ representa as áreas de uma classe que foram perdidas ou convertidas para outra classe no período em estudo, e $\Delta \mathrm{U}_{\text {in }} \geq 0$ denota a área total ocupada por outras classes no cenário "a" que foram convertidas para a classe em análise no cenário "b" (FENG et al. 2011). Se $0<P s<1$, o índice sugere que a mudança naquele tipo de uso e cobertura da terra apresenta tendência de aumentar ou expandir; Ps tendendo a 1 sugere que a classe apresenta predomínio de transição em um único sentido. $P_{s}$ próximo de zero significa que está ocorrendo uma transição de via dupla com expansão limitada, 
caracterizando uma mudança balanceada para aquela classe; se $-1<\mathrm{P}_{\mathrm{s}}<0$, o índice sugere que a mudança na classe apresenta uma tendência decrescente. $P_{s}$ tendendo a -1 sugere que a classe apresenta uma transição dominantemente em um único sentido e a mudança é extremamente desbalanceada (LUO et al. 2008).

Para analisar as mudanças considerando o conjunto de classes de uso e cobertura da terra existentes na bacia hidrográfica, pode ser utilizado o índice de estado e tendência total da mudança $\left(P_{t}\right)$, obtido a partir da equação abaixo (FENG et al. 2011; WANG e WANG, 2013):

$$
P_{t}=\frac{S_{a}}{S_{s}}=\frac{\sum_{1}^{n}\left(\Delta U_{\text {in-i }}-\Delta U_{\text {out }-i}\right)}{\sum_{1}^{n}\left|\Delta U_{\text {in-i }}+\Delta U_{\text {out }-i}\right|} \times 100 \%,\left(S_{s} \neq 0,0 \leq P_{t} \leq 1\right)
$$

Equação 8

Em que $S_{a}$ refere-se à mudança total de uma classe e $S_{s}$ refere-se a mudança em rede.

\section{RESULTADOS}

\subsection{Mudanças no uso e cobertura da terra}

Na tabela 1 apresenta-se a quantificação do uso e cobertura da terra e das mudanças nos quatro cenários estudados.

Tabela 1 - Uso e cobertura da terra na bacia do Arroio Bocarra entre 1985 e 2017

\begin{tabular}{|c|c|c|c|c|c|c|c|}
\hline \multirow{2}{*}{$\begin{array}{c}\text { Ano/ } \\
\text { Período }\end{array}$} & & \multicolumn{6}{|c|}{ Uso e cobertura da terra } \\
\hline & & $\begin{array}{l}\text { Corpo } \\
\text { d'água }\end{array}$ & $\begin{array}{c}\text { Área } \\
\text { florestal }\end{array}$ & Silvicultura & $\begin{array}{c}\text { Cultura } \\
\text { temporária }\end{array}$ & Campestre & $\begin{array}{c}\text { Área } \\
\text { Urbanizada }\end{array}$ \\
\hline \multirow{2}{*}{1985} & Área $\left(\mathrm{km}^{2}\right)$ & 3,64 & 27,59 & 0,98 & 27,84 & 154,9 & 0,13 \\
\hline & $\%$ & 1,69 & 12,83 & 0,46 & 12,94 & 72,02 & 0,06 \\
\hline \multirow{2}{*}{1996} & Área $\left(\mathrm{km}^{2}\right)$ & 5,12 & 20,72 & 0,66 & 40,3 & 148,12 & 0,16 \\
\hline & $\%$ & 2,38 & 9,63 & 0,31 & 18,74 & 68,87 & 0,07 \\
\hline \multirow{2}{*}{2003} & Área $\left(\mathrm{km}^{2}\right)$ & 6,25 & 14,04 & 11,43 & 30,58 & 152,55 & 0,23 \\
\hline & $\%$ & 2,91 & 6,53 & 5,31 & 14,22 & 70,93 & 0,11 \\
\hline \multirow{2}{*}{2017} & Área $\left(\mathrm{km}^{2}\right)$ & 9,2 & 14,99 & 1,95 & 116,16 & 72,53 & 0,25 \\
\hline & $\%$ & 4,28 & 6,97 & 0,91 & 54,01 & 33,72 & 0,12 \\
\hline $\begin{array}{c}1985- \\
1996\end{array}$ & & 0,13 & $-0,62$ & $-0,03$ & 1,13 & $-0,61$ & 0,01 \\
\hline
\end{tabular}


10 | Estudo Da Dinâmica Da Paisagem Na Bacia Hidrográfica Do Arroio Bocarra, Bagé, RS

\begin{tabular}{cccccccc}
$1996-$ & $\begin{array}{c}\text { Taxa de } \\
\text { mudança }\end{array}$ & 0,16 & $-0,95$ & 1,54 & $-1,39$ & 0,62 & 0,03 \\
2003 & $\begin{array}{c}\text { mon- } \\
\left(\mathrm{km}^{2} \text {.ano }{ }^{-1}\right)\end{array}$ & 0,27 & 0,09 & $-0,86$ & 7,78 & $-7,27$ & 0,00 \\
2017 & & & & & & \\
& $\begin{array}{c}\text { Mudança na } \\
\text { ára } \\
\text { ocupada } \\
\text { km² }\end{array}$ & 5,56 & $-12,6$ & 0,97 & 88,32 & $-82,37$ & 0,12 \\
$1985-$ & & & & & & \\
2017 & $\%$ & 152,75 & $-45,67$ & 98,98 & 317,24 & $-53,18$ & 92,31 \\
\hline
\end{tabular}

Fonte: Organização dos autores.

Para complementar a visualização e compreensão dos dados apresentados na tabela 1 apresenta-se o gráfico da dinâmica temporal das classes de uso e cobertura da terra na figura 2 e o padrão espacial do uso e cobertura da terra a figura 2.

Figura 2 - Dinâmica temporal das classes de uso de cobertura da terra da Bacia Hidrográfica do Arroio Bocarra, RS. Fonte: O autor, 2020.

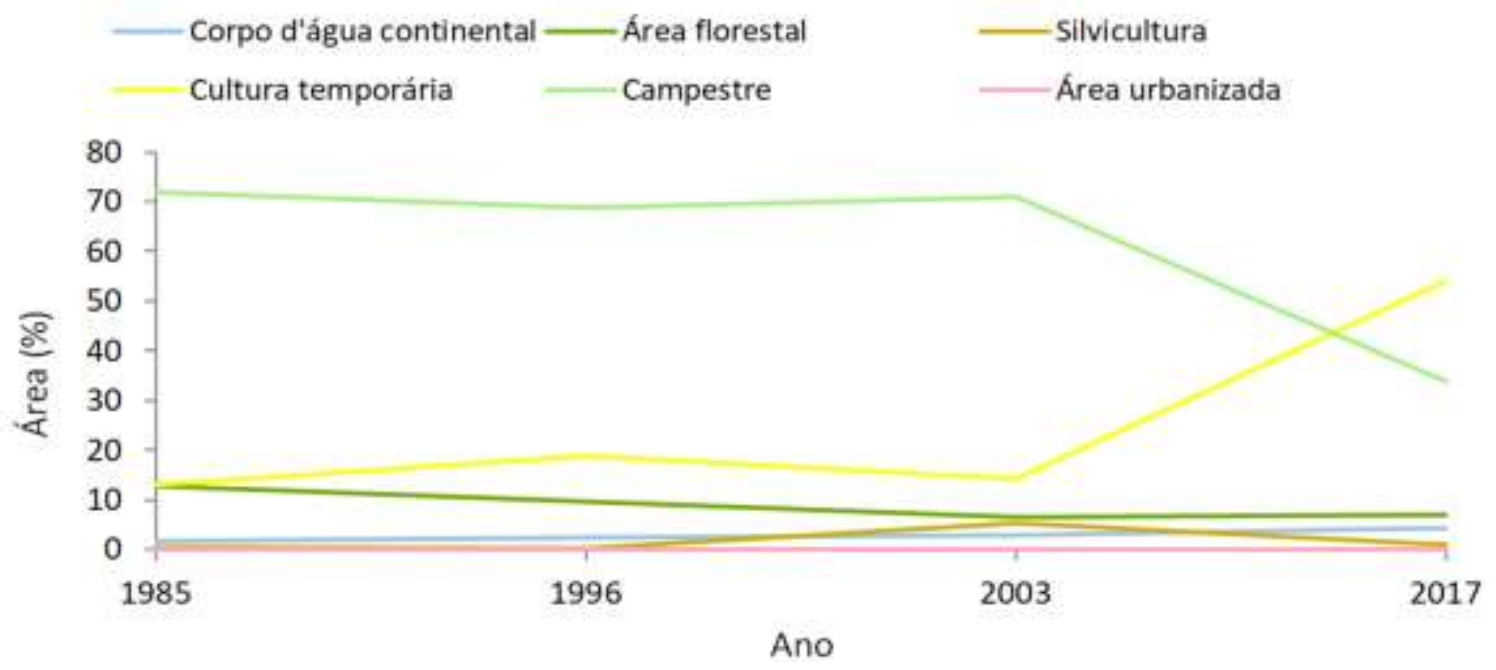

No cenário de 1985 verifica-se o predomínio de áreas campestres, que ocupavam mais de $70 \%$ da área da bacia, enquanto as classes "Área florestal" e "Cultura temporária" ocupavam, juntas, pouco mais de $25 \%$. As áreas destinadas à agricultura concentravam-se na porção central da bacia, enquanto as áreas florestais eram encontradas ao longo dos principais cursos d'água. 
No cenário referente a 1996 detecta-se aumento nas áreas ocupadas por culturas temporárias e redução nas áreas campestres e florestais. Na comparação entre 1985 e 1996 houve redução nas áreas campestres, florestais e de silvicultura, a taxas de 0,61 km².ano-1, 0,62 km².ano-1 e 0,03 km².ano-1, respectivamente. Já as áreas urbanizadas e ocupadas por corpos d'água e culturas temporárias aumentaram a taxas de 0,01 km².ano-1, 0,13 km².ano1 e $1,13 \mathrm{~km}^{2}$.ano-1, respectivamente.

No cenário referente a 2003 verifica-se pequeno aumento nas áreas campestres e de silvicultura e redução pouco significativa nas áreas florestais e destinadas a agricultura quando comparado ao cenário de 1996. Nesse período, as áreas florestais reduziram a uma taxa de 0,95 km².ano-1 e as áreas ocupadas por culturas temporárias a uma taxa de 1,39 $\mathrm{km}^{2}$.ano-1. Já as áreas ocupadas pelas classes "campestre" e "silvicultura" passam a aumentar, a taxas de 0,62 km².ano-1 e 1,54 km².ano-1, respectivamente. As áreas urbanizadas e ocupadas por corpos d'água aumentam.

Entre 2003 e 2017 verifica-se inversão nas tendências de expansão e retração nas classes "campestre" e "cultura temporária". Enquanto as áreas campestres e destinadas a silvicultura reduziram a taxas de 7,27 km².ano-1 e 0,86 km².ano-1, respectivamente, as áreas florestais e as áreas ocupadas por culturas temporárias aumentaram a taxas de 0,09 km².ano-1 e 7,78 km².ano-1, respectivamente. Observa-se ainda continuação da expansão dos corpos d'água, a uma taxa de $0,27 \mathrm{~km}^{2}$.ano-1. Destaca-se o aumento expressivo nas áreas ocupadas por culturas temporárias, que passaram a abranger mais da metade da área da bacia hidrográfica, enquanto as áreas campestres sofreram drástica redução e passaram a ocupar pouco mais de $30 \%$ da área da bacia. Verifica-se ainda redução nas áreas florestais e nas áreas destinadas à silvicultura, além de aumento considerável na área ocupadas pelos corpos d'água (criação de novos reservatórios). 
Figura 3 - Padrão espacial do uso e cobertura da terra na bacia hidrográfica do Arroio Bocarra em 1985, 1996, 2003 e 2017. Fonte: O autor, 2020.
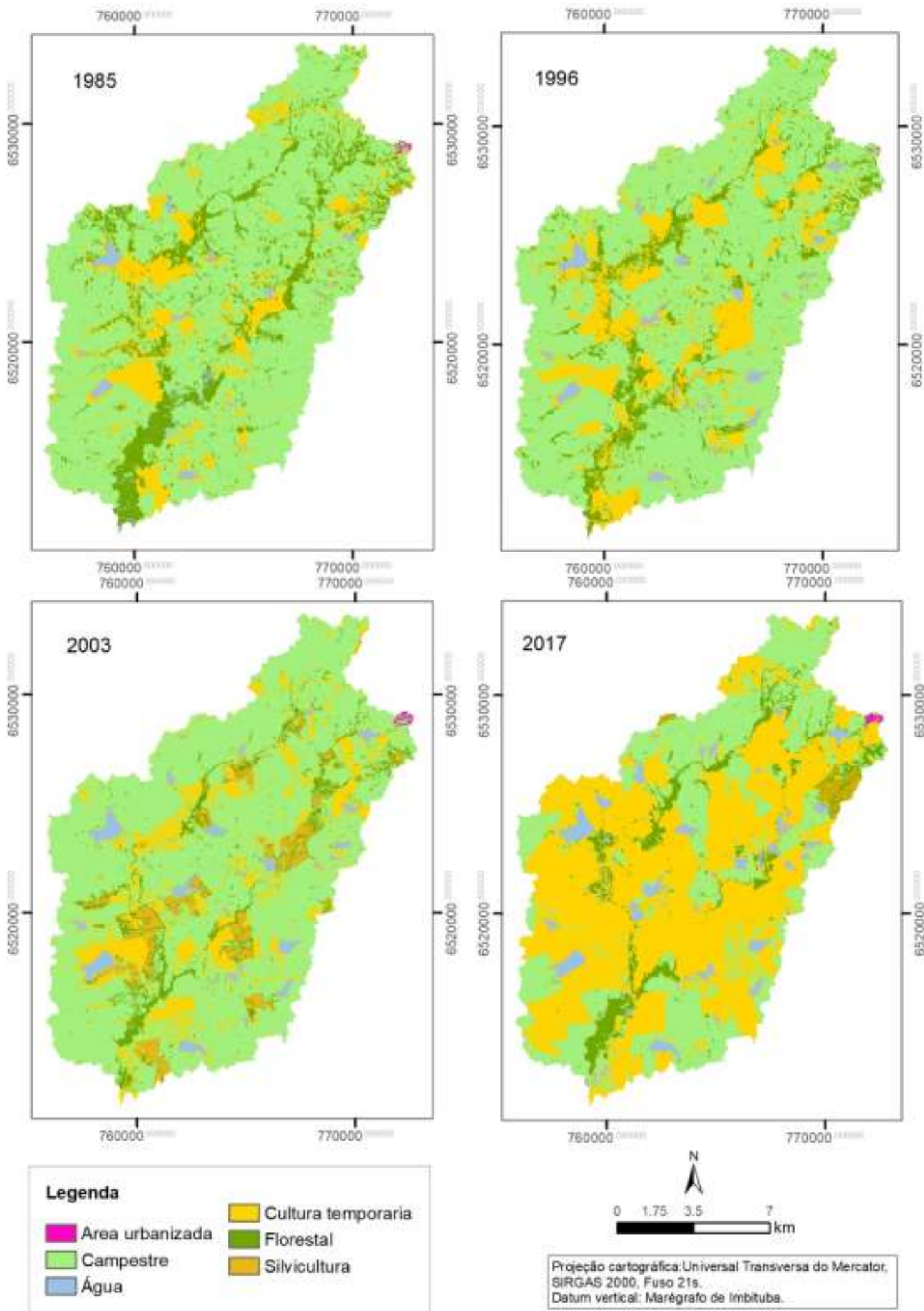
Buscando uma visão mais nítida das mudanças que ocorreram entre 1985 e 2017, apresentam-se na figura 4 as transições e persistências que ocorreram nas classes "Campestre" e "Cultura temporária" nesse período.

Figura 4 - Distribuição espacial das transições e persistências da classe "campestre" e "Cultura temporária" entre 1985 e 2017. Fonte: O autor, 2020.
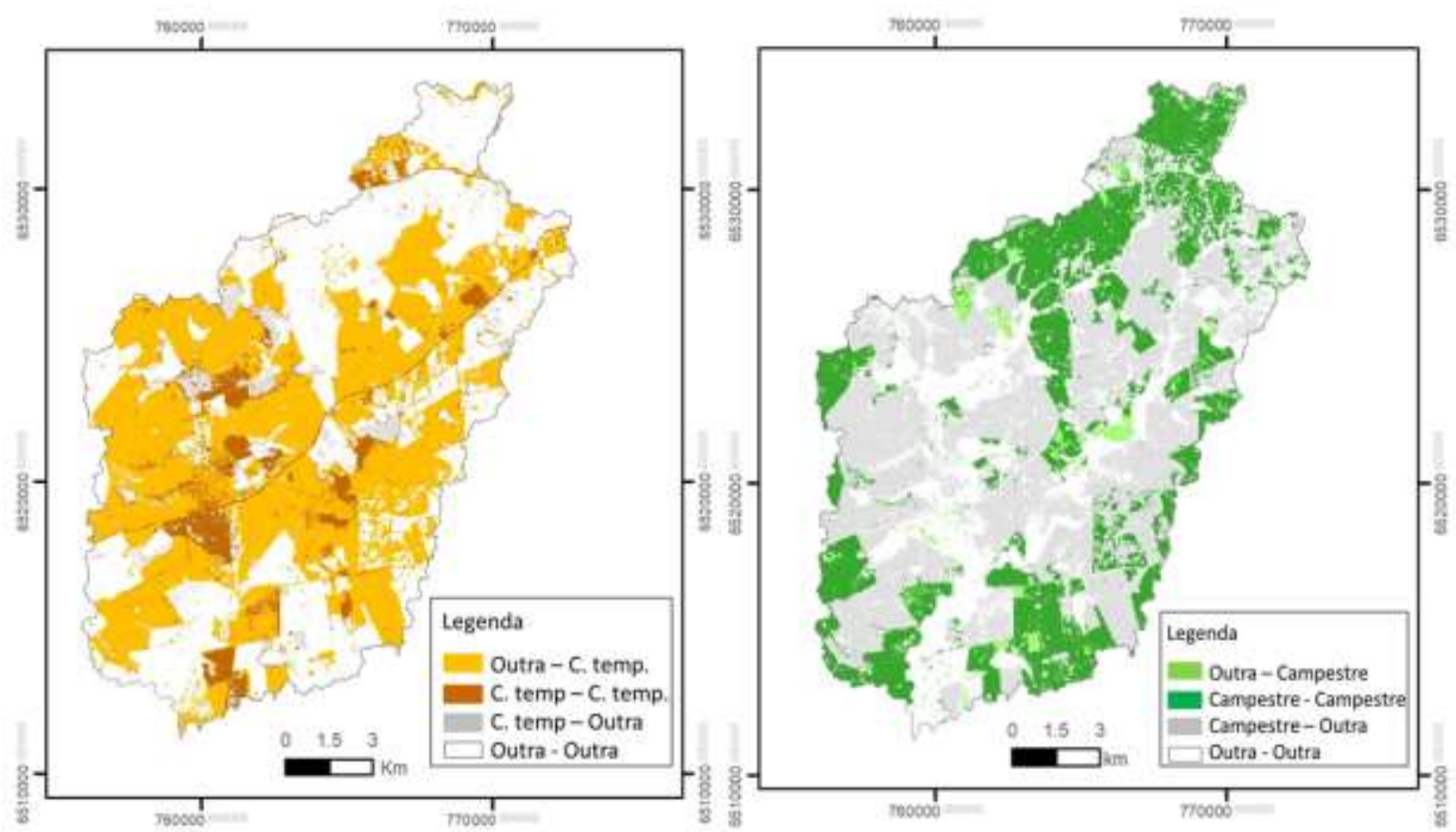

É possível verificar que o avanço da agricultura se deu, em grande parte, em áreas ocupadas por campos em 1985, principalmente nas partes centrais da bacia hidrográfica. Entre 1985 e 2017, as áreas campestres reduziram 53,18\% (82,37 km²). As áreas urbanizadas aumentaram em $92,31 \%\left(0,12 \mathrm{~km}^{2}\right)$, enquanto as áreas ocupadas por culturas temporárias, corpos d'água e silvicultura aumentaram 317,28\% (88,33 $\left.\mathrm{km}^{2}\right), 152,75 \%\left(5,56 \mathrm{~km}^{2}\right)$, e $98,98 \%$ $\left(0,97 \mathrm{~km}^{2}\right)$, respectivamente. Nas áreas de maior altitude e com infra-estrutura de estradas reduzida, a transição campestre-cultura temporária foi menos acentuada.

Na figura 5 apresentam-se as transições e persistências da classe "Área Florestal" entre 1985 e 2017.

É possível verificar, na figura 5, que as áreas florestais se encontram basicamente nas margens dos cursos d'água, como é típico da vegetação da região. Entre 1985 e 2017 houve 
redução de 45,67\% (12,60 km²) nas áreas florestais. Embora tenha ocorrido expansão das áreas florestais sobre áreas campestres em alguns locais, o predomínio foi de redução das áreas florestais, que foram substituídas por áreas campestres e áreas destinadas a agricultura. Essas reduções ocorreram ao longo de toda a bacia hidrográfica, destacando-se as áreas próximas as nascentes (com maior intensidade próximo aos locais em que houve transição campestre-cultura temporária) e também próximo ao exutório. 
Figura 5 - Distribuição espacial das transições e persistências da classe "Área florestal" entre 1985 e 2017. Fonte: O autor, 2020.

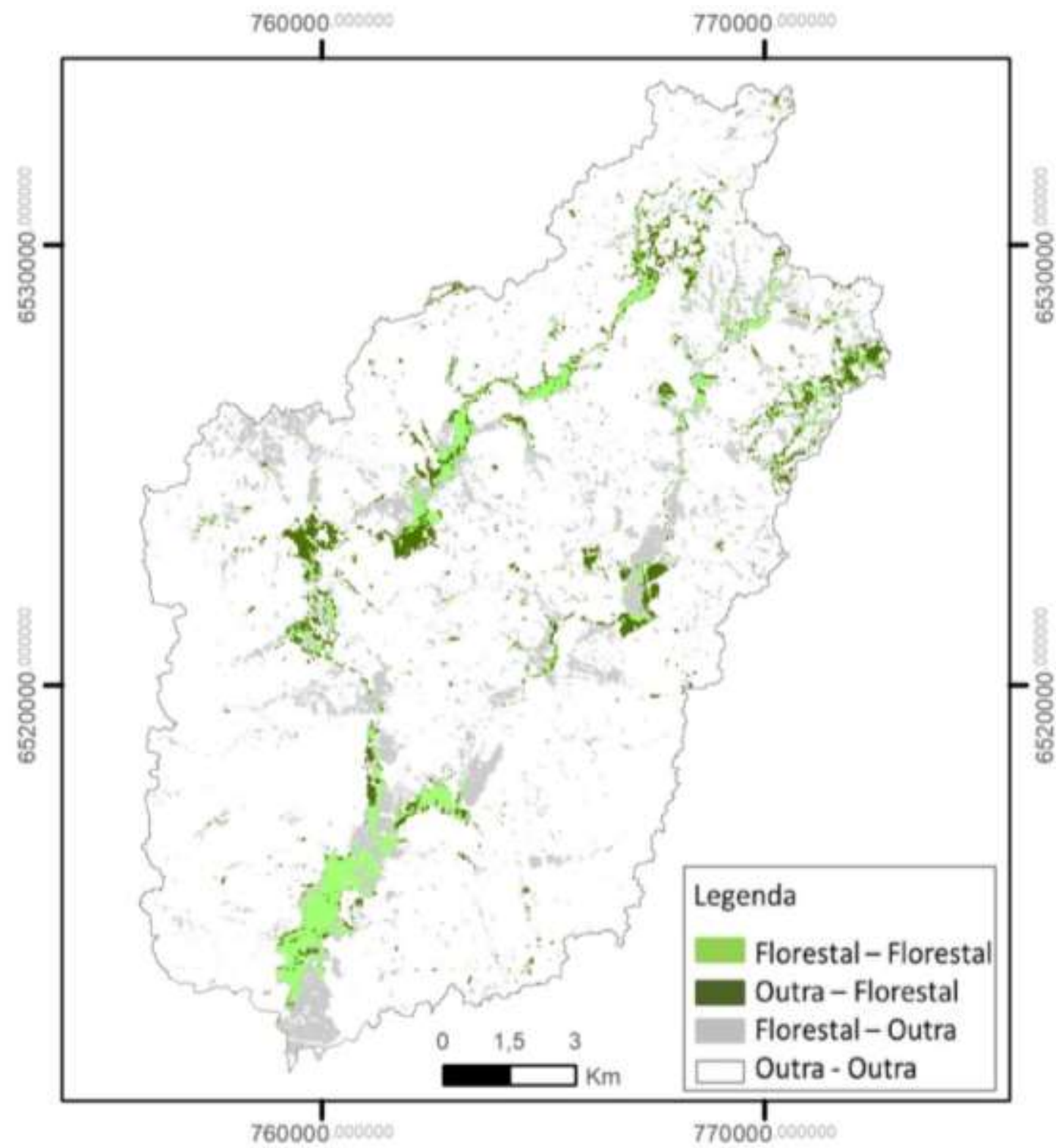

4.2 Tendência das mudanças no uso e cobertura da terra na bacia hidrográfica do Arroio Bocarra

Os valores obtidos para os índices relacionados ao estado e a tendência das mudanças para cada classe de uso e cobertura da terra entre 1985 e 2017 são apresentados 
na tabela 2, enquanto os índices obtidos para o conjunto das classes são apresentados na tabela 3.

Tabela 2 - Índices de estado e tendência das mudanças das classe de uso e cobertura da terra.

\begin{tabular}{ccccccc}
\hline \multirow{2}{*}{ Ano } & Índice & Corpo & Área florestal & Silvicultura & $\begin{array}{c}\text { Cultura } \\
\text { temporária }\end{array}$ & Campestre \\
\hline \multirow{2}{*}{$1985-1996$} & $\mathrm{~N}_{\mathrm{c}}(\%)$ & 40,66 & $-24,90$ & $-32,65$ & 44,76 & $-4,38$ \\
& $\mathrm{~T}_{\mathrm{c}}(\%)$ & 103,30 & 102,39 & 133,67 & 161,21 & 33,89 \\
& $\mathrm{P}_{\mathrm{s}}$ & 0,39 & $-0,24$ & $-0,24$ & 0,28 & $-0,13$ \\
\hline \multirow{2}{*}{$1996-2003$} & $\mathrm{~N}_{\mathrm{c}}(\%)$ & 22,07 & $-32,24$ & 1631,82 & $-24,12$ & 2,99 \\
& $\mathrm{~T}_{\mathrm{c}}(\%)$ & 67,07 & 127,18 & 1736,67 & 113,90 & 40,74 \\
& $\mathrm{P}_{\mathrm{s}}$ & 0,33 & $-0,25$ & 0,94 & $-0,21$ & 0,07 \\
\hline \multirow{2}{*}{$2003-2017$} & $\mathrm{~N}_{\mathrm{c}}(\%)$ & 47,20 & 6,77 & $-82,94$ & 279,89 & $-52,45$ \\
& $\mathrm{~T}_{\mathrm{c}}(\%)$ & 63,12 & 150,92 & 116,60 & 350,10 & 67,26 \\
& $\mathrm{P}_{\mathrm{s}}$ & 0,75 & 0,04 & $-0,71$ & 0,80 & $-0,78$ \\
\hline \multirow{2}{*}{$1985-2017$} & $\mathrm{~N}_{\mathrm{c}}(\%)$ & 152,75 & $-45,67$ & 98,98 & 317,28 & $-53,18$ \\
& $\mathrm{~T}_{\mathrm{c}}(\%)$ & 206,20 & 101,73 & 294,18 & 399,12 & 69,84 \\
& $\mathrm{P}_{\mathrm{s}}$ & 0,74 & $-0,45$ & 0,34 & 0,79 & $-0,76$ \\
\hline
\end{tabular}

Fonte: Organização dos autores.

Tabela 3 - Índices de estado e tendência das mudanças considerando o conjunto das classes de uso e cobertura da terra

\begin{tabular}{lcccc}
\hline & $\mathbf{1 9 8 5 - 1 9 9 6}$ & $\mathbf{1 9 9 6 - 2 0 0 3}$ & $\mathbf{2 0 0 3 - 2 0 1 7}$ & $\mathbf{1 9 8 5 - 2 0 1 7}$ \\
\hline As & 0,07 & 0,08 & 0,41 & 0,44 \\
$S_{s}$ & 0,30 & 0,34 & 0,56 & 0,60 \\
$P_{t}$ & 0,21 & 0,22 & 0,73 & 0,74
\end{tabular}

Fonte: Organização dos autores.

Durante o período 1985-1996 o índice de estado e tendência $P_{t}$ foi 0,21, sugerindo que as mudanças no uso e cobertura da terra foram dominadas por uma transição quase 
balanceada. O índice $\mathrm{P}_{\mathrm{s}}$ para as classes "cultura temporária" e "corpo d'água" foi positivo (aumento de área), apresentando valores de 0,39 e 0,28, respectivamente, sugerindo que a mudança nessas classes foi dominada por uma transição com expansão quase balanceada e de via dupla. O índice Ps para as classes "Área florestal", "Silvicultura" e "Campestre" foi de $-0,24,-024$ e -0,13, respectivamente, sugerindo uma tendência de redução quase balanceada e de via dupla.

No período 1996-2003 o índice de estado e tendência $P_{t}$ foi 0,22, sugerindo que as mudanças continuaram dominadas por uma transição quase balanceada, de via dupla. $\mathrm{O}$ índice Ps para as classes "Área florestal", "Cultura temporária" e "Corpo d'água" foi -0,25, 0,21 e 0,33, respectivamente sugerindo que a mudança em área foi dominada por uma transição balanceada e de via dupla. O índice $P_{s}$ para a classe "Silvicultura" foi de 0,94, sugerindo uma tendência de aumento extremamente desbalanceada, em um sentido (no caso, ganho de área). Para a classe "campestre", o índice $P_{s}$ foi 0,07, sugerindo uma tendência de expansão balanceada e limitada.

No período 2003-2017 o índice de estado e tendência $P_{t}$ foi 0,74, sugerindo que as mudanças no uso e cobertura da terra foram dominadas por uma transição desbalanceada de. O índice $P_{s}$ para as classes "Corpo d'água" e "Cultura temporária" foi de 0,75 e 0,80, respectivamente sugerindo uma tendência de expansão desbalanceada em uma via. O índice $P_{s}$ para a classe "Campestre" e "Silvicultura" foi de -0,71 e -0,78, respectivamente, sugerindo uma tendência de redução extremamente desbalanceada de uma via. $\mathrm{O}$ índice $\mathrm{P}_{\mathrm{s}}$ para a classe "Área florestal" foi de 0,04, sugerindo uma tendência de expansão balanceada e limitada.

\subsection{Mudanças nos padrões de paisagem na bacia hidrográfica do Arroio Bocarra}

As mudanças nos padrões da paisagem foram analisadas com base na fragmentação das classes de uso e cobertura da terra e nos índices de dominância e de diversidade. No gráfico da figura 6 apresenta-se a quantificação da área média dos fragmentos e do índice de fragmentação no período em estudo. 
Figura 6 - Fragmentação na bacia hidrográfica do Arroio Bocarra, RS, entre 1985 e 2017. Fonte: O autor, 2020.

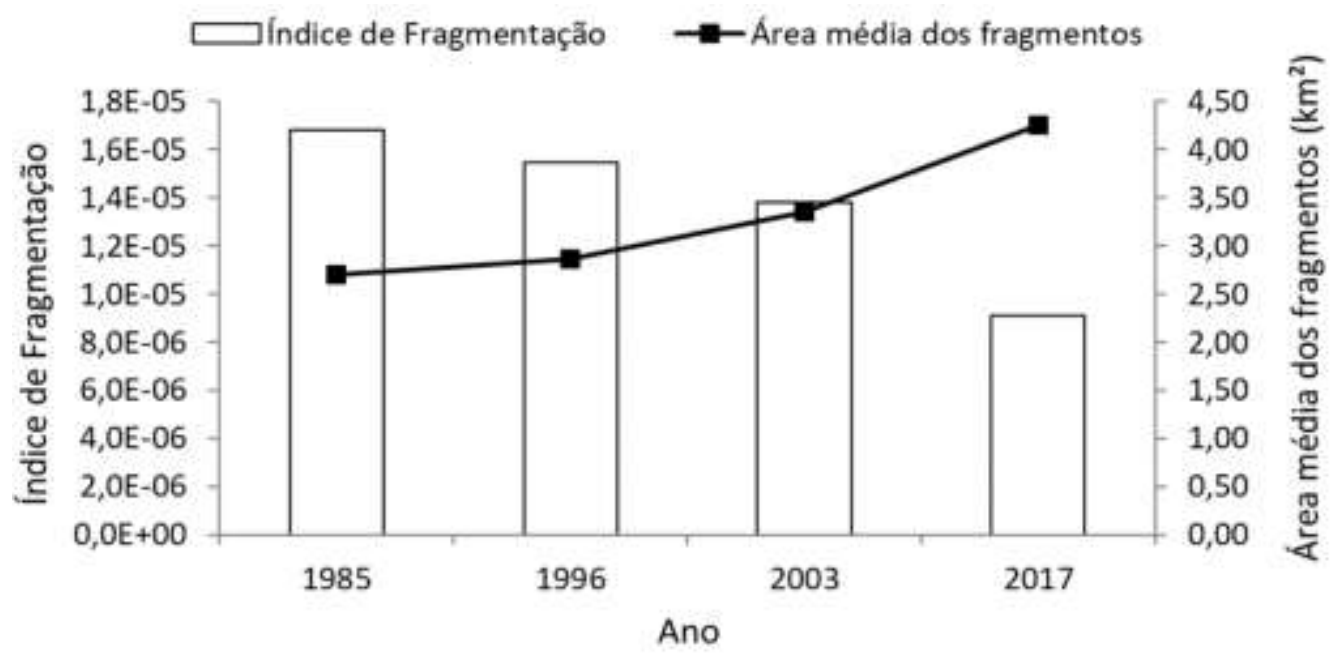

O índice de fragmentação da paisagem diminuiu ao longo de todos os períodos em estudo, apresentando valores da ordem de $1,68.10^{-5}$ em 1985 , reduzindo para $1,54.10^{-5}$ em

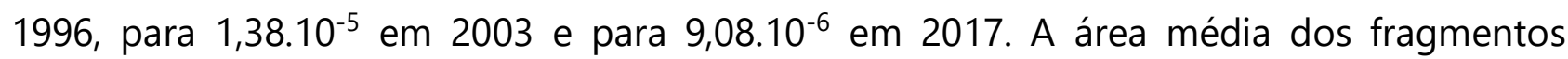
aumentou ao longo de todo o período em estudo: $2,70 \mathrm{~km}^{2}$ em 1985, 2,86km² em 1996, $3,35 \mathrm{~km}^{2}$ em 2003 e 4,25 km² ha em 2017. A redução no índice de fragmentação e o aumento na área média dos fragmentos estão relacionados principalmente à aglomeração de pequenas áreas agrícolas em áreas maiores.

No gráfico da figura 7 apresenta-se a quantificação da área média e do número de fragmentos para cada classe de uso e cobertura da terra no período em estudo. 
Figura 7 - Número e área média de fragmentos na bacia do Arroio Bocarra entre 1985 e 2017. Fonte: O autor, 2020.
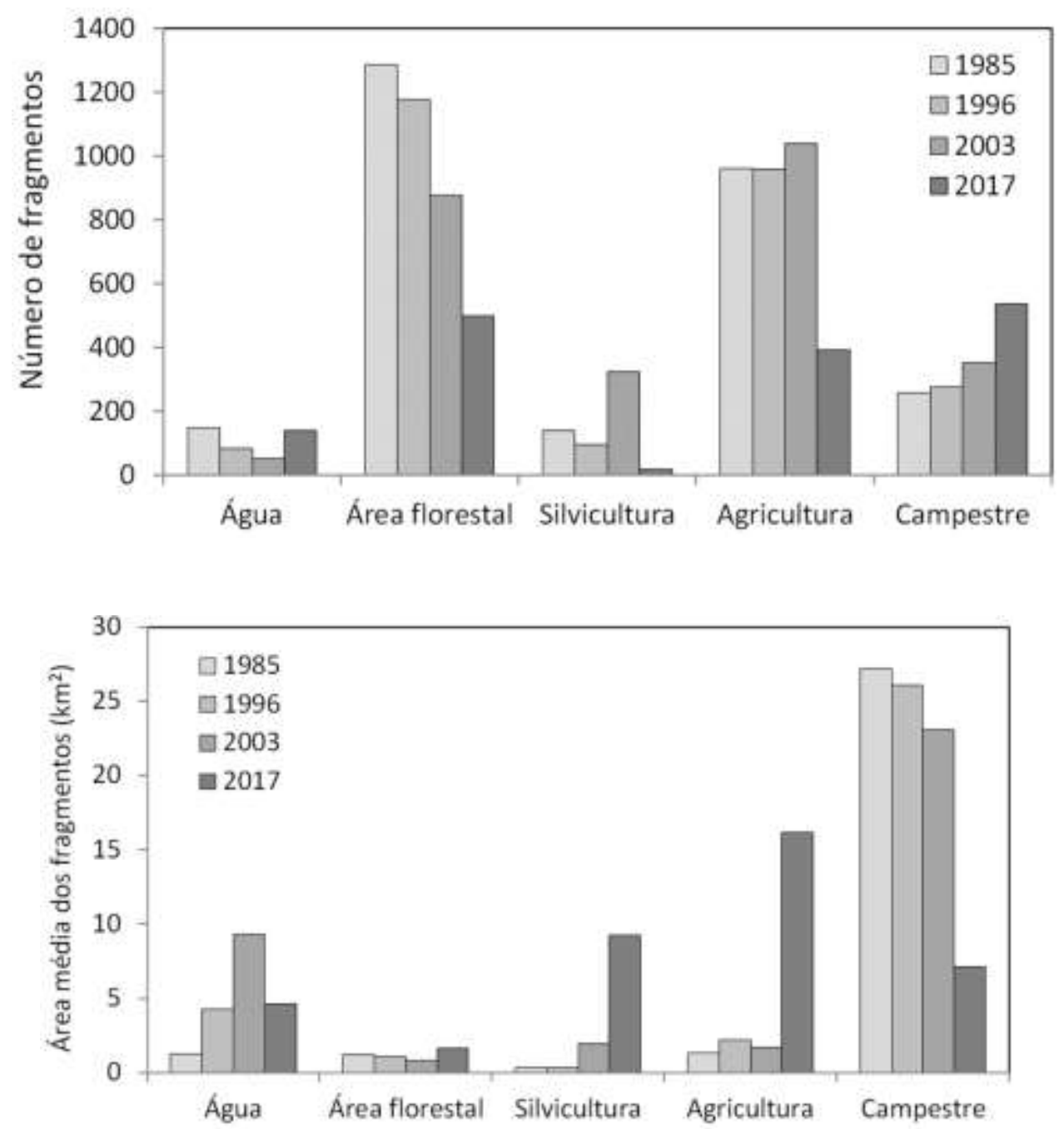

Na figura 7, é possível verificar que no período 1985-1996 houve redução no número de fragmentos para as classes "Área florestal" e "Campestre" e aumento para as classes "Corpo d'água" e "cultura temporária". O valor médio dos fragmentos, em área, aumentou para todas as classes. No período 1996-2003 houve aumento no número de fragmentos para as classes "Silvicultura" e "Campestre". As classes "Corpo d'água", "Área florestal" e "Cultura temporária" apresentaram redução na área dos fragmentos (destaca-se a redução expressiva nas áreas florestais). A área média dos fragmentos aumentou para a classe "Corpos d'água" e "Silvicultura", enquanto as outras classes apresentaram redução. No período 2003-2017 houve considerável redução no número de fragmentos para todas as 
classes. Já a área média dos fragmentos apresentou aumento para as classes "Área florestal", "Silvicultura" e "Cultura temporária" e redução para as classes "Corpo d'água" e "campestre".

O gráfico da figura 8 apresenta a evolução dos índices de diversidade e de dominância na bacia hidrográfica nos cenários em análise.

\section{Figura 8 - Índices de diversidade e de dominância na bacia hidrográfica do Arroio Bocarra} entre 1985 e 2017. Fonte: O autor, 2020.

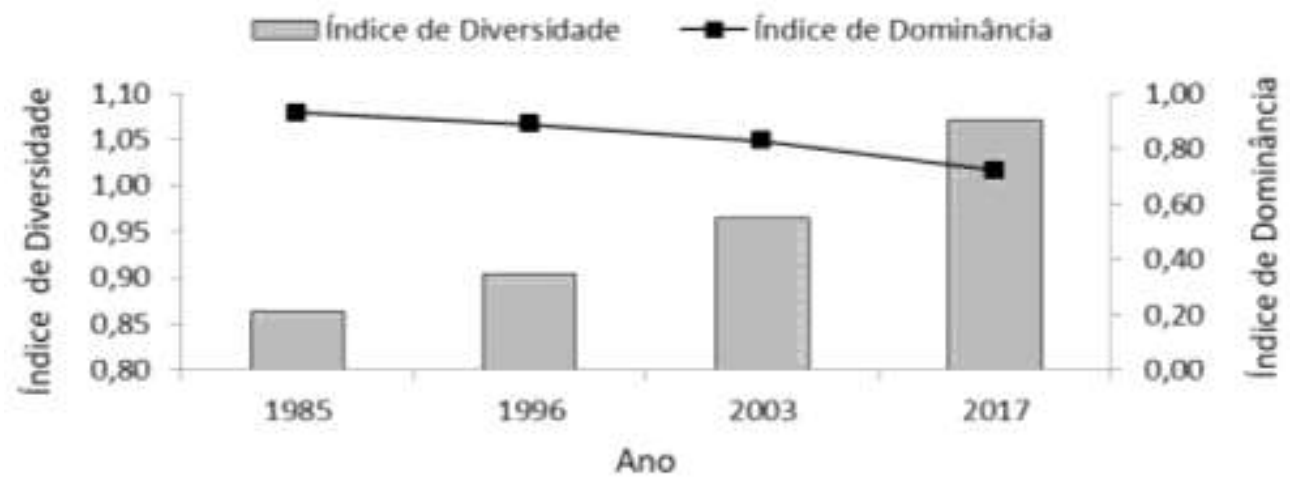

O índice de diversidade da bacia hidrográfica do Arroio Bocarra apresentou redução ao longo do período em estudo, com valores na ordem de 0,93 em 1985, 0,89 em 1996, 0,83 em 2003 e 0,72 em 2017. Já o índice de dominância aumentou ao longo de todo o período, com valores da ordem de 0,86 em 1985, 0,90 em 1996, 0,96 em 2003 e 1,07 em

\section{CONCLUSÕES}

Ao longo do período em estudo, as classes mais representativas em termos de área ocupada foram "Área florestal", "Campestre" e "Cultura temporária". Entre 1985 e 2017 ocorreram alterações consideráveis nos padrões de uso e cobertura da terra da bacia hidrográfica, com redução na vegetação natural (áreas florestais e campestres) e aumento nas áreas destinadas a atividades agrícolas. Alavancado pelo expressivo aumento no cultivo de soja, as áreas ocupadas por culturas temporárias passaram a ocupar a maior parte da bacia hidrográfica em 2017. Essa expansão da agricultura sobre áreas campestres e florestais ocorreu ao longo de toda a extensão da bacia hidrográfica. 
Com relação aos estados e tendências das mudanças do conjunto das classes de uso e cobertura da terra, foi possível verificar a ocorrência de uma transição quase balanceada, de via dupla nos períodos 1 (1985-1996) e 2 (1996-2003). O índice de direção e tendência das classes de uso e cobertura da terra $\left(P_{s}\right)$ mostra transições balanceadas de duas vias em praticamente todas as classes nesses períodos. No período 3 (2003-2017) as mudanças na bacia hidrográfica foram dominadas por uma transição desbalanceada, de uma via. O índice Ps mostra transições desbalanceadas, com grandes perdas na classe "Campestre" e consideráveis ganhos na classe "Cultura temporária", confirmando o avanço das áreas agrícolas sobre as áreas campestres.

As alterações no uso e cobertura da terra que ocorreram ao longo do período em estudo levaram ao aumento do índice de diversidade e redução do índice de dominância, o que pode ser explicado devido ao fato de as áreas campestres estarem dominando a paisagem em 1985 e terem sido, gradualmente, substituídas por áreas agrícolas. A redução das pequenas áreas florestais ao longo dos cursos d'água e a densificação das áreas agrícolas levou à redução do índice de fragmentação e ao aumento da área média dos fragmentos. Analisando a fragmentação para cada classe de uso e cobertura da terra separadamente, verifica-se que os cenários de 1996 e de 2003 apresentavam-se mais fragmentados e que a área média dos fragmentos de cada classe apresentou diferentes tendências de alteração ao longo do período em estudo (por exemplo, a área média dos fragmentos para a classe campestre apresentou redução ao logo do tempo, enquanto para a classe "cultura temporária" houve aumento entre 1985 e 1996, redução entre 1996 e 2003 e um forte aumento entre 2003 e 2017).

Com base nos resultados apresentados é possível afirmar que as mudanças no uso e cobertura da terra tiveram influência direta nas alterações dos padrões de paisagem analisados no estudo.

\section{AGRADECIMENTOS}

O autor do artigo agradece ao CNPQ pela bolsa concedida pelo Programa Institucional de Bolsa de Iniciação Científica (PIBIC/Unipampa/CNPq). 


\section{REFERÊNCIAS}

ALEMAYEHU, F.; TAHA, N.; NYSSEN, J.; GIRMA, A.; ZENEBE, A.; BEHAILU, M.; POESEN, J. The impacts of watershed management on land use and land cover dynamics in Eastern Tigray (Ethiopia). Resources, Conservation and Recycling, v. 53(4), p. 192-198, 2009.

BAIDYA, N.; BHUJU, D.; KANDEL, P. Land use change in buffer zone of Chitwan National Park, Nepal between 1978 and 1999. Ecoprint, v. 16, p. 79-86, 2009.

BEBI, P.; SEIDL, R.; MOTTA, R.; FUHR, M.; FIRM, D.; KRUMM, F.; CONEDERA, M.; GINZLER, C.; WOHLGEMUTH, T.; KULAKOWSKI, D. Changes of forest cover and disturbance regimes in the mountain forests of the Alps. Forest Ecology and Management, v. 388, p. 43-56, 2017.

\section{BRITALDO, S. Modelagem da Dinâmica de Paisagem de uma Região de Fronteira de} Colonização Amazônica. Tese de Doutorado, Escola Politécnica da USP, 1998.

CARPIO, A. J.; OTEROS, J.; TORTOSA, F. S.; GUERRERO-CASADO, J. Land use and biodiversity patterns of the herpetofauna: The role of olive groves. Acta Oecologica, v. 70, p. 103-111, 2016.

CHEN, Y.H.; ZHOU, Y.N.; GE, Y.; AN, R.; CHEN, Y. Enhancing Land Cover Mapping through Integration of Pixel-Based and Object-Based Classifications from Remotely Sensed Imagery. Remote Sensing, v. 10, 77, 2018.

DUAN, C.; PEILI S.; SONG, M.; ZHANG, X.; ZONG, N.; ZHOU, C. Land Use and Land Cover Change in the Kailash Sacred Landscape of China. Sustainability, v. 11, 1788, 2019.

EITER, S.; POTTHOFF, K. Improving the factual knowledge of landscapes: Following up the European Landscape Convention with a comparative historical analysis of forces of landscape change in the Sjodalen and Stølsheimen mountain areas, Norway. Norwegian Journal of Geography, v. 61, p. 145-156, 2007.

FENG, Y.; LUO, G.; LU, L. Effects of land use change on landscape pattern of the Manas River watershed in Xinjiang, China. Environmental Earth Sciences, v. 64, p. 2067-2077, 2011.

FOODY, G.M. Status of land cover classification accuracy assessment. Remote Sensing Environment, v. 80, p. 185-201, 2002.

FREITAS, M.; PABLO MUÑOZ, P.; SANTOS, J.; ALVES, D. Land use and cover change modelling and scenarios in the Upper Uruguay Basin (Brazil). Ecological Modelling, v. 384, p. 128-144, 2018.

IBGE, Instituto Brasileiro de Geografia e Estatística. Manual Técnico de Uso da Terra. Rio de Janeiro, 2013. 
JIMÉNEZ, A.; VILCHEZ, F.; GONZÁLEZ, O.; FLORES, S. Analysis of the Land Use and Cover Changes in the Metropolitan Area of Tepic-Xalisco (1973-2015) through Landsat Images. Sustainability, v. 10, 1860, 2018.

LAMBIN, E. F., GEIST, H. J.; LEPERS, E. (2003). Dynamics of land-use and land-cover change in tropical regions. Annual review of environment and resources, v. 28(1), p. 205-241, 2003.

LANDIS, J.R.; KOCH, G.G. The measurement of observer agreement for categorical data. Biometrics, v.33 (1), p. 159-174, 1977.

LIU, J.; DOU, Y.; BATISTELLA, M.; CHALLIES, E.; CONNOR, T.; FRIIS, C.; MILLINGTON, J.D.; PARISH, E.; ROMULO, C.L.; SILVA, R.F.B. Spillover systems in a telecoupled anthropocene: Typology, methods, and governance for global sustainability. Current Opinion in Environmental Sustainability. V. 33, p. 58-69, 2018.

LUO G.P.; ZHOU C.H.; CHEN X.; LI Y. A methodology of characterizing status and trend of land changes in oases: a case study of Sangong River Watershed, Xinjiang, China. Journal of Environmental Management, v. 88(4), p. 775-783, 2008.

LUO, K.; LI, B.; MOIWO, J. Monitoring Land-Use/Land-Cover Changes at a Provincial Large Scale Using an Object-Oriented Technique and Medium-Resolution Remote-Sensing Images. Remote Sensing, v. 10 (12), 2018.

MCGARIGAL, K.; CUSHMAN, S. A.; ENE, E. (2012). Fragstats v4: Spatial Pattern Analysis Program for Categorical and Continuous Maps (Computer software program produced by the authors at the University of Massachusetts). Amherst, MA: Fragstats.

O'NEILL, R.V.; KRUMMEL, J.R.; GARDNER, R.H.; SUGIHARA, G.; JACKSON, B.; DEANGDLIS, D.L.; MILNE, B.T.; TURNER, M.G.; ZYGMUN,T B.; CHRISTENSEN, S.W.; DALE, V.H.; GRAHAM, R.L. Indices of landscape pattern. Landscape Ecology, v. 1(3), p. 153-162, 1988.

PETZ, K. Mapping and Modelling the Effects of Land Use and Land Management Change on Ecosystem Services: From Local Ecosystems and Landscapes to Global Biomes. Dissertation. Wageningen University, USA, 2014.

PONTIUS, R.G.; SHUSAS, E.; MCEACHERN, M. Detecting importante categorical land changes while accounting for persistence. Agriculture, Ecosystems and Environment, v. 101, p. 251-268, 2004.

TEIXIDO, N.; GARRABOU, J. Spatial pattern quantification of Antarctic benthic communities using landscape indices. Marine Ecology Progress Series, v. 242, p. 1-14, 2002. 
TITEUX, N.; HENLE, K.; MIHOUB, J.B.; REGOS, A.; GEIJZENDORFFER, I.R.; CRAMER, W.; VERBURG, P.H.; BROTONS, L. Biodiversity scenarios neglect future land-use changes. Global Change Biology, v. 22, p. 2505-2515, 2016.

WANG, J.; YANG, X. A Hierarchical Approach to Forest Landscape Pattern Characterization. Environmental Management, v. 49, p. 64-81, 2012.

WANG, S.; WANG, S. Land use/land cover change and their effects on landscape patterns in the Yanqi Basin, Xinjiang (China). Environmental Monitoring Assessment,v. 185, p. 97299742, 2013.

WANG, J.; CHENG, Q.; CHEN, J. A gis and remote sensing-based analysis of land use change using the asymmetric relation analysis method: A case study from the city of hangzhou, China. Mathematical Geosciences, v. 43, p. 435-453, 2011.

YANG, C.; YU, M.; HU, F.; JIANG, Y.; LI, Y. Utilizing cloud computing to address big geospatial data challenges. Computer Environment and Urban System, v. 61, p. 120-128, 2016. 